\title{
A Pulse Wave Velocity Based Method to Assess the Mean Arterial Blood Pressure Limits of Autoregulation in Peripheral Arteries
}

\author{
Ananya Tripathi ${ }^{1 \dagger}$, Yurie Obata ${ }^{1 \dagger}$, Pavel Ruzankin ${ }^{2,3}$, Narwan Askaryar ${ }^{1}$, Dan E. Berkowitz ${ }^{1}$, \\ Jochen Steppan ${ }^{1}$ and Viachaslau Barodka ${ }^{1 *}$
}

${ }^{1}$ Department of Anesthesiology and Critical Care Medicine, Johns Hopkins University School of Medicine, Baltimore, MD, United States, ${ }^{2}$ Sobolev Institute of Mathematics, Novosibirsk, Russia, ${ }^{3}$ Department of Mathematics and Mechanics, Novosibirsk State University, Novosibirsk, Russia

OPEN ACCESS

Edited by:

Agustín Guerrero-Hernández, Center for Research and Advanced Studies of the National Polytechnic Institute (CINVESTAV), Mexico

Reviewed by:

Roland Pittman,

Virginia Commonwealth University, United States

Erik Josef Behringer, Loma Linda University, United States

*Correspondence: Viachaslau Barodka vbarodk1@jhmi.edu

${ }^{\dagger}$ These authors have contributed equally to this work.

Specialty section:

This article was submitted to

Vascular Physiology,

a section of the journal

Frontiers in Physiology

Received: 04 August 2017 Accepted: 13 October 2017 Published: 02 November 2017

Citation:

Tripathi A, Obata Y, Ruzankin P, Askaryar N, Berkowitz DE, Steppan J and Barodka V (2017) A Pulse Wave Velocity Based Method to Assess the Mean Arterial Blood Pressure Limits of Autoregulation in Peripheral Arteries.

Front. Physiol. 8:855

doi: 10.3389/fphys.2017.00855
Background: Constant blood flow despite changes in blood pressure, a phenomenon called autoregulation, has been demonstrated for various organ systems. We hypothesized that by changing hydrostatic pressures in peripheral arteries, we can establish these limits of autoregulation in peripheral arteries based on local pulse wave velocity (PWV).

Methods: Electrocardiogram and plethysmograph waveforms were recorded at the left and right index fingers in 18 healthy volunteers. Each subject changed their left arm position, keeping the right arm stationary. Pulse arrival times (PAT) at both fingers were measured and used to calculate PWV. We calculated $\triangle P A T(\triangle P W V$ ), the differences between the left and right PATs (PWVs), and compared them to the respective calculated blood pressure at the left index fingertip to derive the limits of autoregulation.

Results: $\triangle P A T$ decreased and $\triangle P W V$ increased exponentially at low blood pressures in the fingertip up to a blood pressure of $70 \mathrm{mmHg}$, after which changes in $\triangle \mathrm{PAT}$ and $\triangle \mathrm{PWV}$ were minimal. The empirically chosen $20 \mathrm{mmHg}$ window $(75-95 \mathrm{mmHg})$ was confirmed to be within the autoregulatory limit (slope $=0.097, p=0.56$ ). $\Delta$ PAT and $\Delta$ PWV within a $20 \mathrm{mmHg}$ moving window were not significantly different from the respective data points within the control 75-95 mmHg window when the pressure at the fingertip was between 56 and $110 \mathrm{mmHg}$ for $\triangle P A T$ and between 57 and $112 \mathrm{mmHg}$ for $\triangle \mathrm{PWV}$.

Conclusions: Changes in hydrostatic pressure due to changes in arm position significantly affect peripheral arterial stiffness as assessed by $\triangle P A T$ and $\triangle \mathrm{PWV}$, allowing us to estimate peripheral autoregulation limits based on PWV.

Keywords: limits of autoregulation, pulse wave velocity, pulse arrival time, hydrostatic pressure, peripheral artery

\section{INTRODUCTION}

Various organs are equipped with an autoregulation mechanism in order to maintain constant blood flow as local blood pressure changes (Peterson et al., 2011). These autoregulation mechanisms rely on robust arterial reactivity: as blood pressure decreases, muscular arterioles dilate, and as blood pressure increases, muscular arterioles constrict (Meng and Gelb, 2015). The lower limit of autoregulation represents the pressure at which arteries are maximally dilated and organ perfusion 
becomes pressure dependent. If blood pressure falls below the lower limit of autoregulation, then blood flow decreases. The upper limit of autoregulation represents the pressure at which the arteries are maximally constricted (Meng and Gelb, 2015). If blood pressure goes above the upper limit of autoregulation, then blood flow increases. This physiological mechanism has been demonstrated for the brain, kidney, bone, intestinal tracts, and even the peripheral arteries in the lower extremities (Lassen, 1959; Johnson, 1967; Cupples and Braam, 2007; Vogt et al., 2013).

Limits of autoregulation have been assessed by measuring arterial blood flow velocity during changes of mean arterial blood pressure (Czosnyka et al., 2009). However, it remains challenging to measure the lower limit of autoregulation in humans, especially in vital organs, as this requires inducing potentially dangerously low mean arterial blood pressures, which of course is not ethically acceptable. However, peripheral arteries are routinely exposed to periods of low blood pressures. Raising the arm with respect to the level of the heart is a non-invasive way to decrease local blood pressure by introducing a hydrostatic pressure gradient. Decreasing local blood pressure then leads to a physiologic decrease in wall tension of the corresponding peripheral arteriole. The resultant changes in wall tension elicited by hydrostatic pressure gradients can be quantified precisely since blood density is known and arm length can be measured easily (Butlin et al., 2015).

Measuring arterial blood flow velocity to detect the limits of autoregulation can be done in real time and non-invasively using Doppler ultrasound, a technique which is operator-dependent and time consuming. It has been reported previously that pulse wave velocity (PWV) and the velocity of flow velocity wave transmission are nearly identical (Pai and Shah, 1999). Hence as blood pressure decreases, PWV should have a similar relationship to flow and exhibit a noticeable lower limit of autoregulation.

PWV is a measure of arterial stiffness and depends on the elasticity of the arterial wall (Bramwell and Hill, 1922). The elasticity of the arterial wall in turn depends on both the intrinsic arterial wall composition and wall tension. We have shown previously that PWV increases as MAP increases (Steppan et al., 2014). However, the relationship between PWV and MAP is not linear as PWV increases exponentially at high blood pressures, whereas the changes in PWV are relatively minor when blood pressure is in the normal range. This phenomenon is based on the composition and structure of the arterial wall and its load-bearing components (mainly elastin and collagen) (Steppan et al., 2014). Similar to active blood flow autoregulation, the elastin fibers dampen the changes in blood pressure, thereby maintaining a relatively constant PWV.

We hypothesize that by inducing hydrostatic pressure changes in peripheral arteries by changing arm position, we can evaluate the limits of autoregulation in those arteries via assessing changes in local PWV.

\section{METHODS AND MATERIALS}

\section{Subjects}

This study was approved by the Johns Hopkins Medicine Institutional Review Board (IRB00074229). 18 healthy volunteers were recruited to participate through email or word of mouth following Institutional Review Board approval. Informed oral consent was obtained from all subjects. Inclusion criteria were: healthy adults, aged 18-50 years, and both genders. Exclusion criteria were: subject refusal to participate, known cardiovascular disease of any kind, and pregnancy. After confirming that each subject could participate in the study, each subject self-reported her/his weight, height, age, handedness, and gender.

\section{Study Protocol}

A standard 3 lead electrocardiogram (ECG; Bio Amp FE132, ADInstruments, Australia) was placed on the volunteer to allow for continuous measurement of electrical cardiac activity. We utilized clinically used standard lead locations as suggested by the American Heart Association (AHA) Scientific Statement on Practice Standards for Electrocardiographic Monitoring in Hospital Settings (Drew et al., 2004). A PowerLab analog to digital converter (PowerLab 4/26, ADInstruments, Australia) along with LabChart 8.0 software (LabChart8, Ad Instruments Ltd, Australia) were both used to convert and digitally record the data. Capillary plethysmograph sensors (MLT1020PPG IR Plethysmograph, ADInstruments, Australia) were then placed on the left and right index fingers. The ECG and plethysmograph simultaneously recorded data for each position for $60 \mathrm{~s}$ per position. The subject was seated on a chair with both arm rests at equal heights at the level of the heart. The first position (position A0) was used for a baseline measurement in which the subject had her/his arms resting on the armrests in a horizontal position, at the same height as the subject's heart. The next position (position A1) was identical to position A0 with the difference being that the volunteers left forearm was raised upward, such that subject's forearm was perpendicular to the subject's upperarm, which was resting on the armrest. The subject was then instructed to switch to the up position (position A2), in which the subject raised her/his left arm vertically up above their head while keeping her/his right arm in the same position as it was resting before. The subject then went back to the horizontal position (position B0), with both arms were resting along the same plane as the heart as in position A0. The subject then took her/his left forearm vertically downward, in a half-down position, while keeping her/his upper-arm flat on the armrest (position B1), such as in position A1, just in the opposite direction. The subject did this while holding the right arm in the same position as before. The subject then switched to the final down position (position B2) in which he/she extended her/his left arm all the way down while keeping her/his right arm in the same position as it was resting before (opposite to position A2). After position B2 was completed, the sensors were removed and the subject's blood pressure was recorded using the oscillometric method over the brachial artery. Several lengths were then measured: wingspan (distance from left index finger to right index finger with both arms in $90^{\circ}$ lateral extension), half wingspan on the left side (distance from sternal notch to left index finger with left arm in $90^{\circ}$ lateral extension), the distance between the sternal notch and the axilla on the left arm, the distance between the axilla and the elbow on the left arm, the distance between the elbow and the 
wrist on the left arm, and the distance between the wrist and the left index finger tip on the left arm.

\section{Data Extraction}

Using the collected data, the pulse arrival time (PAT) to both the left and the right index fingertip was calculated by automated algorithm as the time delay between each R-wave peak on the ECG waveform and the first positive inflection on the plethysmograph tracing for both the left and the right side.

\section{Calculations}

PWV for both the left (Equation 1) and right (Equation 2) sides were determined using the measured hemi-span (sternal notch to tip of the index finger) lengths divided by the PAT.

$$
\begin{aligned}
P W V_{\text {Left }} & =\frac{d_{\text {wingspan }}}{2 P A T_{\text {Left }}} \\
P W V_{\text {Right }} & =\frac{d_{\text {wingspan }}}{2 P A T_{\text {Right }}}
\end{aligned}
$$

We also calculated the difference in PAT values ( $\triangle \mathrm{PAT}$ ) between the left and right side and the difference in PWV $(\triangle \mathrm{PWV})$ between the left and right side at each position keeping right arm at the level of the heart (Equations 3 and 4).

$$
\begin{aligned}
\triangle P A T & =P A T_{\text {Left }}-P A T_{\text {Right }} \\
\triangle P W V & =P W V_{\text {Left }}-P W V_{\text {Right }}
\end{aligned}
$$

The hydrostatic blood pressure changes were quantified by multiplying the density of blood ( $\rho=1,060 \mathrm{~kg} / \mathrm{m} 3)$, gravity related acceleration $\left(g=9.81 \mathrm{~m} / \mathrm{s}^{2}\right)$, and the height of the fluid column (d) (Equation 5). To convert the hydrostatic pressure from Pascals to $\mathrm{mmHg}$, the obtained value was multiplied by a conversion factor of 0.0075 (Butlin et al., 2015).

$$
\text { Hydrostatic pressure }=0.0075 \rho g d
$$

For our analysis, we quantified the maximum pressure change at the tip of the finger. Hydrostatic pressures calculated for the up positions ( $\mathrm{A} 1$ and $\mathrm{A} 2$ ) were defined as negative; those calculated for the down positions (B1 and B2) were positive. To calculate the total blood pressure (accounting for both systemic and hydrostatic pressure), the mean arterial pressure (MAP), defined by Equation (6), was added to the hydrostatic pressure (Equation 7).

$$
\begin{aligned}
M A P & =D B P+([S B P-D B P] / 3) \\
\text { Calculated pressure } & =\text { Hydrostatic pressure }+ \text { MAP }
\end{aligned}
$$

\section{Statistical Analysis}

Based on the results of the previous study, we estimated that a sample of 3 subjects would provide adequate power [ $80 \%$ power for a mean (SD) difference of $42.7 \mathrm{~ms}(11.0 \mathrm{~ms})$ ] for the change in PAT between horizontal and up arm position at an alpha level of 0.05 in a two-sided paired $t$-test (Foo et al., 2005). Based on the results of our previous study, we estimated that a sample of 18 subjects would provide adequate power [ $80 \%$ power for a mean (SD) difference of $8.78 \mathrm{~ms}(12.40 \mathrm{~ms})$ ] for the change in PAT between horizontal and down arm position at an alpha level of 0.05 in a two-sided paired $t$-test (Obata et al., 2017).

Paired $t$-tests were used to compare the PATs for each arm between the initial horizontal position (position A0) and the horizontal position after arm raise (position B0) and to compare the PAT and PWV values measured at different left arm positions to the respective values of the right arm, which was constantly kept at the level of the heart. A repeated measure one-way analysis of variance (ANOVA) test was used with the Dunnett's multiple comparisons test to check for differences between PAT, $\triangle \mathrm{PAT}, \mathrm{PWV}$ and $\triangle \mathrm{PWV}$ at each position. We performed a non-linear regression model to assess the relationship between hydrostatic blood pressure change at the tip of the finger and the resultant change in PAT between left and right arm $(\triangle \mathrm{PAT})$, the relationship between the calculated blood pressure at the tip of the finger and the $\triangle \mathrm{PAT}$, and the relationship between the calculated blood pressure at the tip of the finger and $\triangle \mathrm{PWV}$. These analyses were performed with GraphPad Prism version 6.0 (GraphPad Software, San Diego, California, USA). All tests were two-sided. A $p$-value less than 0.05 was considered to be statistically significant.

To detect the point of inflection of $\triangle \mathrm{PAT}$ or $\triangle \mathrm{PWV}$ vs. calculated pressure at the left fingertip relationship, we first created a smoothed curve by applying a LOESS (locally weighted scatter-plot smoother)-smoothed filter to all observed data points. The inflection point of the curve is the point which is maximally distant from the line connecting the curve's ends (Lepeschkin and Surawicz, 1953). The method is robust with respect to the observations' perturbations and is invariant with respect to scaling of the axes. We used the standard LOESS function from the $\mathrm{R}$ package ( $\mathrm{R}$ foundation for Statistical Computing, Vienna, Austria) with values of the span parameter $0.2,0.4$, and 0.75 .

To detect the limits of autoregulation we compared the distribution of $\triangle \mathrm{PAT}$ and $\triangle \mathrm{PWV}$ observations in $\pm 10 \mathrm{mmHg}$ moving windows to the respective distribution within a known autoregulation range and to the right of the inflection point by the two-sided Mann-Whitney test. To confirm that the empirically chosen control range of calculated blood pressure is in fact within the limits of autoregulation, we calculated the linear regression slope and checked to see if the slope was statistically different from 0 . Slopes not different from 0 indicate that the data points within control range are within the autoregulation limit as they show consistent PWV values despite changes in blood pressure. The relationship between the $p$-values of a Mann-Whitney test and blood pressure at the fingertip was plotted. The pressure values at which the plots cross the $p$-value of 0.05 ( $5 \%$ level) were considered to be the lower and higher limits of autoregulation.

\section{RESULTS}

Demographic characteristics and blood pressure values are summarized in Table 1. Each position's average PAT of the left and right arm, $\triangle \mathrm{PAT}$, and PWV is summarized in Table 2 . The 
mean age of the volunteers was approximately 32 years and ranged from 18 to 42 years. At rest, the average systolic blood pressure (SBP) was $115.3 \pm 24.5 \mathrm{mmHg}$ and the average diastolic blood pressure (DBP) was $68.6 \pm 14.5 \mathrm{mmHg}$.

\section{PAT Analysis}

The PAT at positions A0 and B0 were compared using a $t$-test to determine if they were significantly different between the two horizontal readings. The average left PAT values at position A0 were not significantly different from the average left PAT values at position B0 ( $p=0.508)$; similarly, the average right PAT at position A0 was not significantly different from the average right PATs at position $\mathrm{B} 0(p=0.064)$.

The left PATs for each position were compared to their respective horizontal right PATs (Supplementary Figure 1). Leftsided PATs were significantly higher than right-sided PATs at position $\mathrm{A} 1(p=0.0002)$, position $\mathrm{A} 2(p<0.0001)$, and position B0 $(p=0.0003)$.

Left PATs at each position were compared to the left PAT at position A0 (Supplementary Figure 2A). Relative to the left

TABLE 1 | Cohort demographics summary (Mean \pm SD).

\begin{tabular}{lc}
\hline & Mean \pm SD ( $\mathbf{n}$ 18) \\
\hline Age [years] & $32.2 \pm 6.9$ \\
Weight [kg] & $66.7 \pm 14.0$ \\
Height [cm] & $169.9 \pm 6.9$ \\
SBP [mmHg] & $115.3 \pm 24.5$ \\
DBP [mmHg] & $68.6 \pm 14.5$ \\
MAP [mmHg] & $84.1 \pm 17.2$ \\
Wingspan [cm] & $170.7 \pm 8.4$ \\
Half wingspan [cm] & $85.3 \pm 4.2$ \\
d sternalnotch-fingertip [left; cm] & $85.3 \pm 4.2$ \\
d sternalnotch-axilla [left; cm] & $20.1 \pm 1.7$ \\
d axilla-elbow [left; cm] & $22.8 \pm 2.0$ \\
d elbow-wrist [left; cm] & $24.3 \pm 2.0$ \\
d wrist-fingertip [left; cm] & $18.1 \pm 1.8$
\end{tabular}

$S D$, Standard deviation; SBP, Systolic blood pressure; DBP, Diastolic blood pressure; MAP, Mean arterial pressure.
PAT at position A0, the left PAT values were significantly higher at position A1 $(p=0.005)$ and position A2 $(p=0.0001)$ and significantly lower at position B2 $(p=0.003)$. The right PAT values were compared to the right PATs at position A0 (Supplementary Figure 2B). The right PATs were significantly higher at position A2 than those at position A0 $(p=0.023)$, despite only minimal changes. $\triangle$ PATs at each position were compared to the $\triangle \mathrm{PAT}$ values at position A0 (Supplementary Figure $2 \mathrm{C}$ ). Relative to the $\triangle \mathrm{PAT}$ at position $\mathrm{A} 0$, the $\triangle \mathrm{PAT}$ values were significantly higher at position $\mathrm{A} 1(p=0.005)$ and position A2 $(p=0.0001)$.

\section{PWV Analysis}

Left-sided PWV values for each position were compared to their respective horizontal right-sided PWV values (Supplementary Figure 3). Left-sided PWVs were significantly lower than the right-sided values at position A1 $(p<0.0001)$, position A2 $(p<$ $0.0001)$, and position $\mathrm{B} 0(p=0.0003)$.

PWV values on the left were compared to the left PWV values at position A0 (Supplementary Figure 4A). The left side PWV values at position A0 were significantly higher than the left side PWV values at position A1 $(p=0.002)$ and position A2 $(p=0.0001)$. The left side PWV values at position A0 were significantly lower than the left side PWV values at position B2 $(p=0.002)$. Right side PWV values were compared to right side PWV values at position A0 (Supplementary Figure 4B). The right values at position $\mathrm{A} 0$ were significantly higher than those at position $\mathrm{A} 2(p=0.011) . \triangle \mathrm{PWV}$ values were compared to $\triangle \mathrm{PWV}$ s at position A0 (Supplementary Figure $4 \mathrm{C}$ ). Relative to those at position $\mathrm{A} 0, \triangle \mathrm{PWV}$ was significantly lower at position A1 $(p=0.004)$ and position A2 $(p=0.0001)$.

\section{Pressure Analysis}

The hydrostatic pressure was calculated using (Equation 5) (Table 3). The convention implemented for hydrostatic pressure was such that if the left arm was either in position A1 or A2that is, half-up or fully up-then the hydrostatic pressures were considered to be negative; conversely, if the left arm was in position $\mathrm{B} 1$ or $\mathrm{B} 2$, in the half-down or complete down position, then the hydrostatic pressures were considered to be positive. The pressure at the fingertip for each position was calculated

TABLE 2 | Measured and calculated data summary (Mean \pm SD).

\begin{tabular}{|c|c|c|c|c|c|c|}
\hline & AO & A1 & A2 & BO & B1 & B2 \\
\hline $\mathrm{PAT}_{\text {Left }}[\mathrm{ms}]$ & $218.6 \pm 24.2$ & $229.5 \pm 26.4$ & $264.8 \pm 37.6$ & $220.0 \pm 21.1$ & $215.5 \pm 22.7$ & $209.4 \pm 22.6$ \\
\hline PAT $_{\text {Right }}[\mathrm{ms}]$ & $214.8 \pm 24.1$ & $215.6 \pm 24.8$ & $211.5 \pm 24.3$ & $212.7 \pm 23.5$ & $213.2 \pm 24.4$ & $212.2 \pm 24.1$ \\
\hline$\triangle \mathrm{PAT}[\mathrm{ms}]$ & $3.8 \pm 9.1$ & $14.1 \pm 12.4$ & $53.8 \pm 22.8$ & $7.2 \pm 6.8$ & $2.4 \pm 7.2$ & $-1.9 \pm 9.5$ \\
\hline$P W V_{\text {Left }}[\mathrm{m} / \mathrm{s}]$ & $3.9 \pm 0.4$ & $3.8 \pm 0.5$ & $3.3 \pm 0.5$ & $3.9 \pm 0.3$ & $4.0 \pm 0.4$ & $4.1 \pm 0.4$ \\
\hline PWV $W_{\text {Right }}[\mathrm{m} / \mathrm{s}]$ & $4.0 \pm 0.4$ & $4.0 \pm 0.4$ & $4.1 \pm 0.4$ & $4.0 \pm 0.4$ & $4.0 \pm 0.4$ & $4.1 \pm 0.4$ \\
\hline$\Delta \mathrm{PWV}[\mathrm{m} / \mathrm{s}]$ & $-0.1 \pm 0.2$ & $-0.2 \pm 0.2$ & $-0.8 \pm 0.3$ & $-0.1 \pm 0.1$ & $0.0 \pm 0.1$ & $0.0 \pm 0.1$ \\
\hline
\end{tabular}

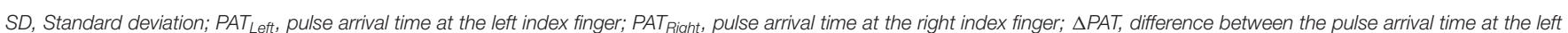

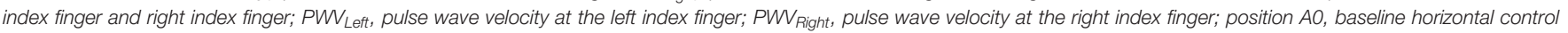

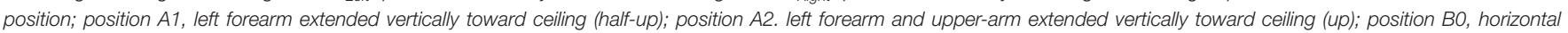

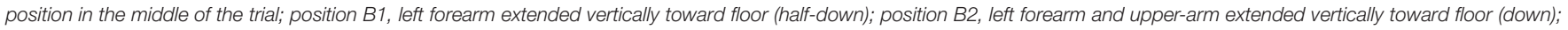
$\mathrm{ms}$, milliseconds; $\mathrm{m} / \mathrm{s}$, meters per second. 
TABLE 3 | Pressure data summary (Mean \pm SD).

\begin{tabular}{lcc}
\hline Position & Hydrostatic pressure $[\mathrm{mmHg}]$ & Actual pressure [mmHg] \\
\hline A0 & $0.0 \pm 0.0$ & $84.1 \pm 17.2$ \\
A1 & $-33.1 \pm 2.0$ & $51.0 \pm 17.2$ \\
A2 & $-50.8 \pm 2.9$ & $33.3 \pm 16.9$ \\
B0 & $0.0 \pm 0.0$ & $84.1 \pm 17.2$ \\
B1 & $33.1 \pm 2.9$ & $117.2 \pm 17.5$ \\
B2 & $50.8 \pm 2.9$ & $134.9 \pm 18.0$
\end{tabular}

SD, Standard deviation; Position A0, baseline horizontal control position; position A1, left forearm extended vertically toward ceiling (half-up); position A2, left forearm and upperarm extended vertically toward ceiling (up); position BO, horizontal position in the middle of the trial; position B1, left forearm extended vertically toward floor (half-down); position $B 2$, left forearm and upper-arm extended vertically toward floor (down).

using Equation (6) (Table 3) and the resulting values were plotted against $\triangle$ PAT (Figure 1A). The average pressure values at the fingertips were then calculated and plotted against the average $\triangle \mathrm{PAT}$ values (Figure 1B). A non-linear one-phase regression model was generated for the average pressure at the fingertips vs. $\triangle \mathrm{PAT}$, yielding (Equation 8 ). The regression model had a coefficient of determination of 0.98 , indicating a strong fit for the data.

$\triangle P A T=6.78 \times 10^{2} \times e^{(-0.08 \times \text { pressure at the fingertips })}+2.14$

The $\triangle \mathrm{PWV}$ values were also compared to the calculated pressure at the fingertips (Figure 1C); the average $\triangle \mathrm{PWVs}$ were plotted against the average pressure at the fingertips at their respective positions (Figure 1D). A non-linear one-phase regression model was generated, yielding Equation (9). This regression model had a coefficient of determination of 0.96 .

$$
\Delta P W V=-7.58 \times e^{(-0.07 \times \text { pressure at the fingertips })}-0.03
$$

\section{Estimation of the Inflection Point}

The inflection point for $\triangle \mathrm{PAT}$ over pressure relationship was detected at 70.67, 70.26, and $71.36 \mathrm{mmHg}$ for the LOESS function span parameter of $0.2,0.4$, and 0.75 respectively (Figure $2 \mathrm{~A}$ ). The inflection point for $\triangle \mathrm{PWV}$ over the pressure relationship was detected at 70.55, 69.02, and $70.61 \mathrm{mmHg}$ for the LOESS function span parameter of $0.2,0.4$, and 0.75 respectively (Figure $2 \mathbf{B}$ ). This means that the window with the lowest regression slope within the autoregulation limit will be located at pressures above 70-71 $\mathrm{mmHg}$.

\section{Estimation of the Control Blood Pressure Window within the Limits of Autoregulation}

We chose a $\pm 10 \mathrm{mmHg}$ window to the right of the inflection point, empirically choosing $75-95 \mathrm{mmHg}$. The estimated regression slope for all data points within the $75-95 \mathrm{mmHg}$ window was 0.097 with $p=0.69$, indicating that it is not statistically different from the regression slope 0 and the 75-95 $\mathrm{mmHg}$ window is in fact within limits of autoregulation. We chose a second window of $80-100 \mathrm{mmHg}$, with the estimated regression slope for all data points within this window being 0.25 with $p=0.36$, indicating that it is not statistically different from a regression slope 0 and the $80-100 \mathrm{mmHg}$ window is within the limits of autoregulation.

\section{Estimation of the Limits of Regional Autoregulation}

The $\triangle$ PAT data within a $\pm 10 \mathrm{mmHg}$ moving window compared to the $\triangle$ PAT data within a control window ranging from 75 to $95 \mathrm{mmHg}$ was significant when blood pressures were below $56 \mathrm{mmHg}$ or above $110 \mathrm{mmHg}$ (Figure 3A). Similarly the $\triangle \mathrm{PWV}$ data within $\pm 10 \mathrm{mmHg}$ moving window compared to the $\triangle \mathrm{PWV}$ data within a control window ranging from 75 to $95 \mathrm{mmHg}$ was significant when blood pressure was below 57 $\mathrm{mmHg}$ and above $112 \mathrm{mmHg}$. (Figure 3B). Changing the control window from $75-95 \mathrm{mmHg}$ to $80-100 \mathrm{mmHg}$ did not change the results. Hence, the lower limit of autoregulation was detected at $56 \mathrm{mmHg}$ with the $p$-value graph derived from $\triangle$ PAT over the calculated blood pressure at the tip of finger relationship (Figure 3A) and at $57 \mathrm{mmHg}$ for the $p$-value graph derived from $\triangle \mathrm{PWV}$ over blood pressure at the tip of finger relationship (Figure 3B). The upper limit of autoregulation was detected at $110 \mathrm{mmHg}$ on the $p$-value graph derived from $\triangle \mathrm{PAT}$ over the pressure relationship (Figure 3A) and at $112 \mathrm{mmHg}$ on the $p$ value graph derived from $\triangle \mathrm{PWV}$ over the pressure relationship (Figure 3B).

\section{DISCUSSION}

In our study, we confirmed that changes in hydrostatic pressure due to changes in arm position significantly affect peripheral PWV and PAT in healthy subjects. In addition, our data suggests the existence of autoregulation in the peripheral arteries of the arm. The average calculated MAP in the study subjects was $84 \mathrm{mmHg}$. The average length of the arm was $85 \mathrm{~cm}$, which corresponds to an average $66 \mathrm{mmHg}$ decrease in pressure due to hydrostatic effects when the arm is up. Hence, the effective distending pressure at the tip of the fingers is $18 \mathrm{mmHg}(84$ $\mathrm{mmHg}-66 \mathrm{mmHg}$ ) in the arm up position and $150 \mathrm{mmHg}$ $(84 \mathrm{mmHg}+66 \mathrm{mmHg})$ in arm down position. As blood pressure at the tip of finger drops below $56 \mathrm{mmHg}$, we found sudden changes in both $\triangle \mathrm{PAT}$ or $\triangle \mathrm{PWV}$ compared to $\triangle \mathrm{PAT}$ or $\triangle \mathrm{PWV}$ at a known autoregulation range of $75-95 \mathrm{mmHg}$, which is consistent with the theory that the vasculature operates on distending pressures below the lower limit of autoregulation. We chose a control distending blood pressure window on the horizontal part of the $\triangle \mathrm{PAT} / \triangle \mathrm{PWV}$ vs. blood pressure at left fingertip graph, to the right of the inflection point, which was 70 $\mathrm{mmHg}$. A range of 75-95 $\mathrm{mmHg}$ was chosen based on published data on the lower limits of autoregulation in the brain and the kidneys (Carlström et al., 2015; Scheeren and Saugel, 2017). We performed the same analysis using different ranges (e.g., 80$100 \mathrm{mmHg}$ ) and observed the same results for the limits of autoregulation. Furthermore, if a particular range of distending blood pressures is within the limits of autoregulation, the specific range should have a slope close to 0 . The slope of the regression 


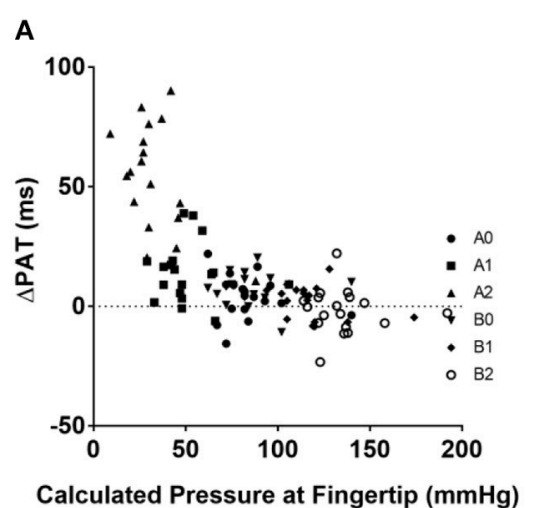

C

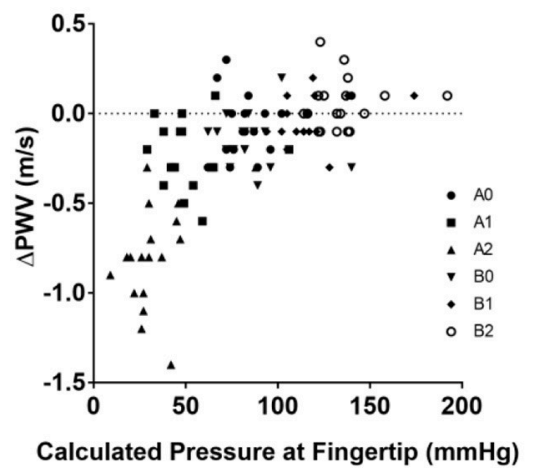

B

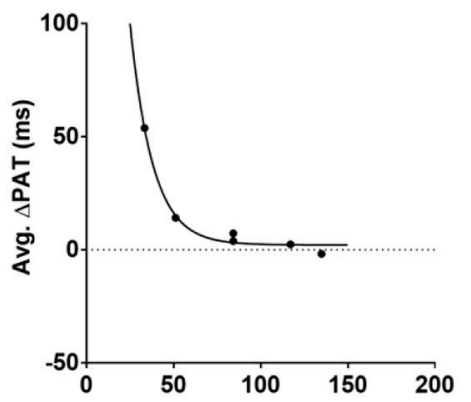

Avg. Calculated Pressure at Fingertip $(\mathrm{mmHg})$

D

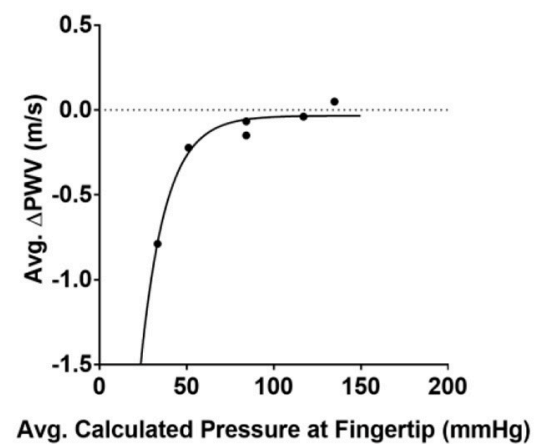

FIGURE 1 | Relationships between blood pressure at the tip of finger and $\triangle$ PAT and $\triangle \mathrm{PWV}$. Calculated pressure values compared to $\triangle \mathrm{PAT}$ and $\triangle \mathrm{PWV}$ values. (A) Calculated blood pressure at the tip of finger for each individual subject at each position and $\Delta$ PAT. (B) Average blood pressure at the tip of finger and average $\triangle$ PAT values with the generated non-linear regression model. (C) Calculated blood pressure at the tip of finger for each individual subject at each position and $\triangle$ PWV values. (D) Average blood pressure at the tip of finger and average $\triangle \mathrm{PWV}$ values with the generated non-linear regression model. $\triangle \mathrm{PAT}$, difference between the pulse arrival time at the left index finger and right index finger; $\triangle \mathrm{PWV}$, difference between the pulse wave velocity at the left index finger and right index finger; ms, milliseconds; $\mathrm{m} / \mathrm{s}$, meters per second.

analysis for the 75-95 $\mathrm{mmHg}$ range was $0.097(p=0.69)$ and for the $80-100 \mathrm{mmHg}$ range $0.25(p=0.36)$. As blood pressure at the tip of finger increased above $112 \mathrm{mmHg}$, we saw changes in both $\triangle \mathrm{PAT}$ and $\triangle \mathrm{PWV}$ compared to those at a known autoregulation range of $75-95 \mathrm{mmHg}$, which is consistent with the theory that the vasculature operates on distending pressures above the upper limit of autoregulation. Using our noninvasive approach, we were able to establish PWV based limits of peripheral artery autoregulation (56-112 mmHg) in human peripheral arteries of the upper extremity. Intriguingly they are very close to the known blood flow based limits of autoregulation described for the brain and kidneys. Thus, the peripheral arteries of the arm could potentially be used as a window into non-invasive assessment of responses in the arterial system to changes in blood pressure and establishing at least the lower limit of autoregulation in individual subjects.

From our data, it is evident that a low distending pressure results in a profound decrease in peripheral arteries PWV in healthy individuals. Interestingly, a notable change in the slope of both $\triangle \mathrm{PAT}$ and $\triangle \mathrm{PWV}$ over the distending blood pressure occurs at $70 \mathrm{mmHg}$. We did not observe such a notable change in the slope at high blood pressures, likely as lowering the arm cannot result in pressures high enough to overcome the upper limit of autoregulation in all subjects.

In an elegant study using ultrasonographic and Doppler techniques, Eiken and Kölegård, showed that indeed changes in intravascular pressure in the range of $75-275 \mathrm{mmHg}$ lead to significant changes in arterial stiffness of peripheral arteries as evident by changes in flow and diameter (Eiken and Kölegård, 2004). Notably, they found that there were greater changes in the flow of the arm arteries compared to the arteries of the leg for the same changes in pressures above $160 \mathrm{mmHg}$. This indicates that the peripheral arterioles especially in the arm are sensitive to changes in distending pressure and present autoregulation behavior. The blood flow at the lowest pressure in their experiment $(70 \mathrm{mmHg})$ did not change from the flow at $160 \mathrm{mmHg}$ suggesting that they were not able to detect the lower limit of autoregulation in the experiments.

A study by Foo et al. examined pulse transit time (PTT) at different limb positions (Foo et al., 2005). They found that there were significant PTT changes in the limb that underwent positional change, relative to a baseline control value. When 

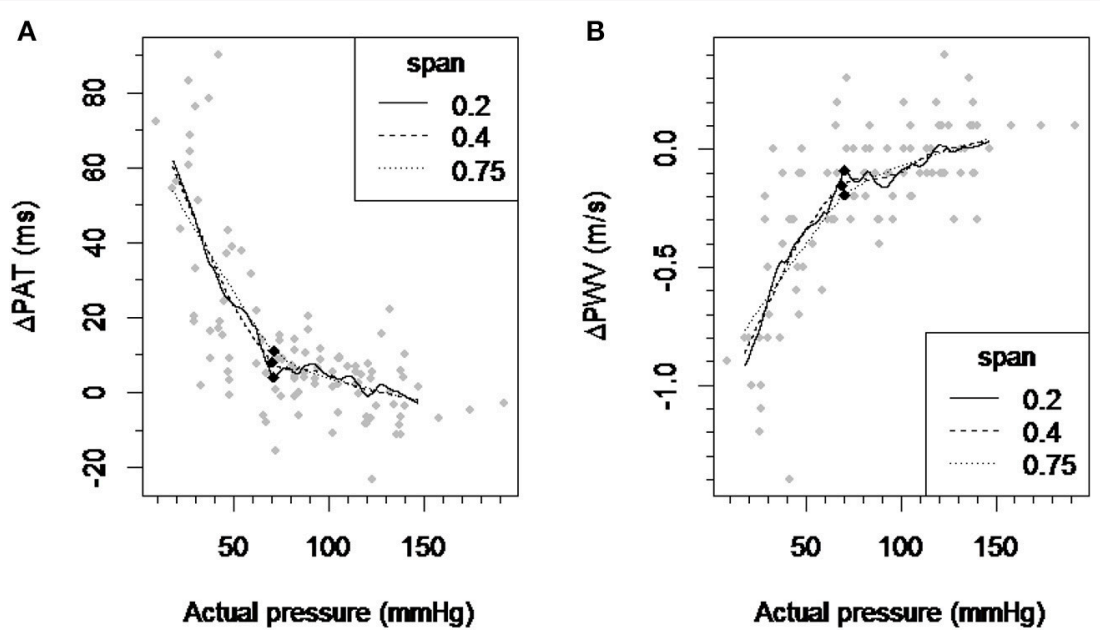

FIGURE 2 | Detection of the inflection point of the $\triangle \mathrm{PAT}$ and $\triangle \mathrm{PWV}$ over pressure relationship. (A) A scatter plot of $\triangle \mathrm{PAT}$ vs. pressure and smoothed curves by applying the LOESS-smoothed filter to all observations with the span parameter values of $0.2,0.4$, and 0.75 . (B) A scatter plot of $\triangle P W V$ vs. pressure and smoothed curves by applying the LOESS-smoothed filter to all observations with the span parameter values of $0.2,0.4$, and 0.75 . Each black square indicates the inflection point which maximally distant from the line connecting the curve's ends. $\triangle \mathrm{PAT}$, difference between the pulse arrival time at the left index finger and right index finger; $\triangle \mathrm{PWV}$, difference between the pulse wave velocity at the left index finger and right index finger; $\mathrm{ms}, \mathrm{milliseconds} ; \mathrm{m} / \mathrm{s}$, meters per second.
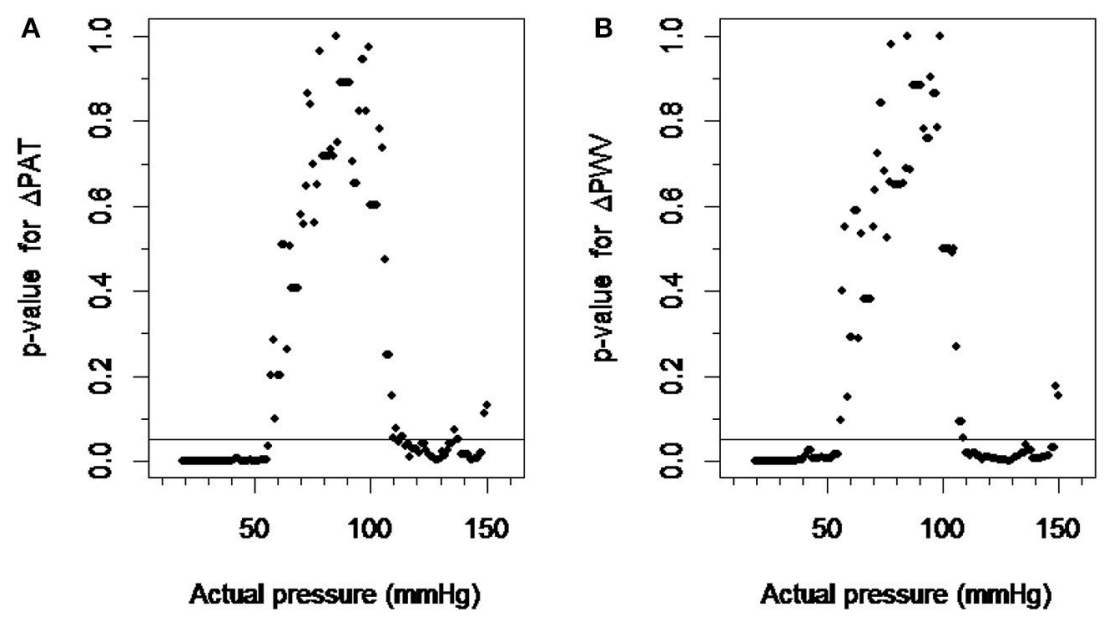

FIGURE 3 | Detection of the limits of autoregulation. (A) The scatter plot between $p$-values derived from $\triangle$ PAT over pressure relationship and actual pressure. (B) The scatter plot between $p$-values derived from $\triangle \mathrm{PWV}$ over pressure relationship and actual pressure. The pressure values at which the plots cross the $p$-value of 0.05 were considered the lower and higher limits of autoregulation. $\triangle \mathrm{PAT}$, difference between the pulse arrival time at the left index finger and right index finger; $\triangle \mathrm{PWV}$,

difference between the pulse wave velocity at the left index finger and right index finger.

the limb was vertically raised, PTT increased; this is consistent with our data. The authors attributed this increase in PTT to changes in hydrostatic pressure and regulation mechanisms within the limbs. They concluded that PTT is indicative of local circulatory responses, suggesting the existence of an autoregulatory mechanism within the arteries of the arm.

In our previous study, we showed that PAT changes significantly depend on the site of measurement (ear lobe, index finger, or big toe) (Obata et al., 2017). In that study, the volunteers changed their positions from supine to sitting to standing. Changing position from supine to standing introduces hydrostatic pressure gradients in the thoracic, abdominal, and peripheral arteries of the lower extremities. However, the observed changes in PAT and PWV did not allow to distinguish hydrostatic effects between the central and peripheral circulation. Moreover, changing the entire body position from supine to standing introduces significant global hemodynamic effects (such as heart rate and blood pressure changes) which affected arterial wall tension and PWV independent of hydrostatic blood pressure changes. This did not allow us to distinguish hydrostatic blood pressure changes from hemodynamically induced changes in the arterial wall. 
The current study design enabled us to assess the effect of hydrostatic blood pressure changes specifically on peripheral arteries and to minimize the global changes in the hemodynamic state. Changing the position of only one arm introduced minimal hemodynamic effects compared to postural changes of the whole body. The right arm was consistently kept at the level of the heart to serve as an intrinsic control, canceling out any potential systemic hemodynamic effects. Thus, we quantified the relationship between distending pressure (MAP plus hydrostatic pressure changes due to changes in arm position) and $\triangle \mathrm{PAT}$ with $\triangle \mathrm{PWV}$. Importantly, it allowed us to calculate the limits of autoregulation in a peripheral artery in a simple and non-invasive way. It would be intriguing to see if there is a correlation between limits of autoregulation in a peripheral artery and the brain.

\section{Limitations}

Our study has several limitations. For MAP, we used only one single baseline blood pressure reading at position A0. There is a possibility that MAP would change with changes in the arm position. However, we believed that those changes will be minimal. Inflating the blood pressure cuff itself will induce local changes by squeezing the arm arteries, which we wanted to avoid. We used the peak of the R-wave on the ECG as the reference point for PAT and substituted pulse transit time with PAT for PWV calculations. The pre-ejection phase in healthy subjects is about $35 \mathrm{~ms}$ (Biering-Sørensen et al., 2016), whereas the PAT is in range of $150-300 \mathrm{~ms}$, thus PAT overestimates true pulse transit time by $15 \%$. However, we calculated $\triangle$ PAT between the left and right arm, which would cancel out the pre-ejection time and the time pulse wave spend in central aorta. To determine the limits of autoregulation, we used each position data point for every subject; this means, however, that there were a limited number of data points for each subject. Data points at angle increments for each subject would be more adequate in order to generate a smoother and more comprehensive model, which

\section{REFERENCES}

Biering-Sørensen, T., Mogelvang, R., de Knegt, M. C., Olsen, F. J., Galatius, S., and Jensen, J. S. (2016). Cardiac time intervals by tissue doppler imaging Mmode: normal values and association with established echocardiographic and invasive measures of systolic and diastolic function. PLOS ONE 11:e0153636. doi: 10.1371/journal.pone.0153636

Bramwell, J. C., and Hill, A. V. (1922). The velocity of the pulse wave in man. Proc. R. Soc. Lond. B 93, 298-306. doi: 10.1098/rspb.1922.0022

Butlin, M., Hathway, P. J., Kouchaki, Z., Peebles, K., and Avolio, A. P. (2015). A simplified method for quantifying the subject-specific relationship between blood pressure and carotid-femoral pulse wave velocity. Conf. Proc. IEEE Eng. Med. Biol. Soc. 2015, 5708-5711. doi: 10.1109/EMBC.2015.7319688

Carlström, M., Wilcox, C. S., and Arendshorst, W. J. (2015). Renal autoregulation in health and disease. Physiol. Rev. 95, 405-511. doi: 10.1152/physrev.00042.2012

Cupples, W. A., and Braam, B. (2007). Assessment of renal autoregulation. Am. J. Physiol. Renal. Physiol. 292, F1105-F1123. doi: 10.1152/ajprenal.00194.2006

Czosnyka, M., Brady, K., Reinhard, M., Smielewski, P., and Steiner, L. A. (2009). Monitoring of cerebrovascular autoregulation: facts, myths, and missing links. Neurocrit. Care 10, 373-386. doi: 10.1007/s12028-008-9175-7

Drew, B. J., Califf, R. M., Funk, M., Kaufman, E. S., Krucoff, M. W., Laks, M. M., et al. (2004). Practice standards for electrocardiographic monitoring in hospital settings: an American heart association scientific would yield more precise limits of autoregulation in individual subjects. Finally, the limits of autoregulation in our experiments are based on PWV instead of flow velocity. Without simultaneous measurements of Doppler flow velocity we cannot guarantee that PWV based limits of autoregulation are same as flow velocity based.

\section{CONCLUSIONS}

In conclusion, our study shows that changes in hydrostatic pressure due to positional changes of the arm significantly affects peripheral arterial stiffness. This allows us to estimate the limits of regional autoregulation based on PWV.

\section{AUTHOR CONTRIBUTIONS}

VB conceived the study and developed the protocol; AT, YO, NA, and VB collected data; AT, YO, PR, NA, and VB analyzed and interpreted the data; AT, YO, PR, DB, JS, and VB drafted and revised the manuscript. All authors read and approved the final version of the manuscript.

\section{FUNDING}

This work was supported by StAAR (Stimulating and Advancing ACCM Research) grant to VB from the Department of Anesthesiology and Critical Care Medicine, The Johns Hopkins University.

\section{SUPPLEMENTARY MATERIAL}

The Supplementary Material for this article can be found online at: https://www.frontiersin.org/articles/10.3389/fphys. 2017.00855/full\#supplementary-material

statement from the councils on cardiovascular nursing, clinical cardiology, and cardiovascular disease in the young: endorsed by the international society of computerized electrocardiology and the american association of criticalcare nurses. Circulation 110, 2721-2746. doi: 10.1161/01.CIR.0000145144. 56673.59

Eiken, O., and Kölegård, R. (2004). Comparison of vascular distensibility in the upper and lower extremity. Acta Physiol. Scand. 181, 281-287. doi: 10.1111/j.1365-201X.2004.01291.x

Foo, J. Y. A., Wilson, S. J., Williams, G. R., Harris, M.-A., and Cooper, D. M. (2005). Pulse transit time changes observed with different limb positions. Physiol. Meas. 26, 1093-1102. doi: 10.1088/0967-3334/26/6/018

Johnson, P. C. (1967). Autoregulation of Blood Flow in the Intestine. Gastroenterology 52, 435-441.

Lassen, N. A. (1959). Cerebral blood flow and oxygen consumption in man. Physiol. Rev. 39, 183-238.

Lepeschkin, E., and Surawicz, B. (1953). The duration of the Q-U interval and its components in electrocardiograms of normal persons. Am. Heart J. 46, 9-20. doi: 10.1016/0002-8703(53)90237-3

Meng, L., and Gelb, A. W. (2015). Regulation of cerebral autoregulation by carbon dioxide. Anesthesiology 122, 196-205. doi: 10.1097/ALN.0000000000000506

Obata, Y., Ong, Q. J., Magruder, J. T., Grichkevitch, H., Berkowitz, D. E., Nyhan, D., et al. (2017). Noninvasive assessment of the effect of position and exercise on pulse arrival to peripheral vascular beds in healthy volunteers. Front. Physiol. 8:47. doi: $10.3389 /$ fphys.2017.00047 
Pai, R. G., and Shah, P. M. (1999). Relationship between the pulse wave and the flow velocity wave and their propagation velocities in the arterial system: implications for the assessment of regional physical properties of the arterial beds. Int. J. Angiol. 8, 127-130. doi: 10.1007/BF01616831

Peterson, E. C., Wang, Z., and Britz, G. (2011). Regulation of cerebral blood flow. Int. J. Vascular Med. 2011, 1-8. doi: 10.1155/2011/823525

Scheeren, T. W. L., and Saugel, B. (2017). Journal of clinical monitoring and computing 2016 end of year summary: monitoring cerebral oxygenation and autoregulation. J. Clin. Monit. Comput. 31, 241-246. doi: 10.1007/s10877-017-9980-7

Steppan, J., Sikka, G., Jandu, S., Barodka, V., Halushka, M. K., Flavahan, N. A., et al. (2014). Exercise, vascular stiffness, and tissue transglutaminase. J. Am. Heart Assoc. 3:e000599. doi: 10.1161/JAHA.113.000599

Vogt, S., Venjakob, A. J., Stöckl, K., Tischer, T., Jost, P. J., Imhoff, A. B., et al. (2013). Evidence of an autoregulatory mechanism of regional bone blood flow at hypotension. Arch. Orthop. Trauma Surg. 133, 1233-1241. doi: 10.1007/s00402-013-1801-y

Conflict of Interest Statement: The authors declare that the research was conducted in the absence of any commercial or financial relationships that could be construed as a potential conflict of interest.

Copyright (C) 2017 Tripathi, Obata, Ruzankin, Askaryar, Berkowitz, Steppan and Barodka. This is an open-access article distributed under the terms of the Creative Commons Attribution License (CC BY). The use, distribution or reproduction in other forums is permitted, provided the original author(s) or licensor are credited and that the original publication in this journal is cited, in accordance with accepted academic practice. No use, distribution or reproduction is permitted which does not comply with these terms. 